\title{
Measuring the neutrino mass ordering and other oscillation parameters with KM3NeT-ORCA
}

\author{
Antoine Kouchner* \\ University Paris Diderot - AstroParticule and Cosmology \\ E-mail: kouchnereapc.univ-paris7.fr \\ João Coelho \\ University Paris Diderot - AstroParticule and Cosmology \\ E-mail: jcoelho@apc.in2p3.fr
}

on behalf of the KM3NeT Collaboration

\begin{abstract}
ORCA (Oscillations Research with Cosmics in the Abyss) is the low-energy branch of KM3NeT, the next generation underwater Cherenkov neutrino detector in the Mediterranean. Its primary goal is to resolve the long-standing unsolved question of whether the neutrino mass ordering is normal or inverted by measuring matter oscillation effects with atmospheric neutrinos. The ORCA design foresees a dense configuration of KM3NeT detection units, optimised for studying the interactions of neutrinos in seawater at low $(<100 \mathrm{GeV})$ energies. To be deployed at the French KM3NeT site, ORCA's multi-PMT optical modules will exploit the excellent optical properties of deep seawater to accurately reconstruct both cascade (mostly electron neutrinos) and track events (mostly muon neutrinos) with a few $\mathrm{GeV}$ of energy. This contribution discusses the potential of the ORCA detector both in neutrino mass ordering studies and in obtaining new constraints on other key parameters such as $\theta_{23}$. New sensitivity studies of exotic oscillation phenomena such as sterile neutrinos and non-standard interactions are also presented.
\end{abstract}

35th International Cosmic Ray Conference - ICRC2017

10-20 July, 2017

Bexco, Busan, Korea

${ }^{*}$ Speaker. 


\section{Introduction}

The main objectives of the KM3NeT Collaboration are the discovery and subsequent observation of high-energy neutrino sources in the Universe [1] and the determination of the mass ordering of neutrinos. To meet this second objective, the KM3NeT Collaboration is building a dense deepsea detector dubbed ORCA (Oscillation Research with Cosmics in the Abyss) as part of a larger network of deep-sea neutrino telescopes in the Mediterranean Sea, in order to study the oscillations of atmospheric neutrinos.

Oscillation experiments are not sensitive to the absolute value of neutrino masses but provide measurements of the squared-mass splittings $\Delta m_{i j=1 ; 2 ; 3}^{2}=m_{j}^{2}-m_{i}^{2}$. Oscillation parameters are extracted from global fits of available data, but several fundamental properties remain to be determined: the octant of $\theta_{23}$ (i.e. whether $\theta_{23}$ is greater or smaller than $\pi / 4$ ), the value of $\delta_{C P}$ and the neutrino mass ordering (NMO). A normal ordering (NO) scenario would apply in the case where $m_{1}<m_{2}<m_{3}$, while the ordering $m_{3}<m_{1}<m_{2}$ is referred to as inverted (IO).

The ORCA strategy for determining the NMO is to make accurate studies of the oscillation patterns of atmospheric neutrinos as a function of their energies and incident angles (i.e their path through the Earth). Depending on the NMO these patterns can differ because of matter effects that modify the oscillation probabilities in the few-GeV domain, which impact the effective values of the mixing angles and squared mass splittings [2]. Although the matter effects are opposite for antineutrinos and neutrinos, the cross-section of neutrinos is about twice larger than for anti-neutrinos at the energies relevant for a NMO measurement and the flux of atmospheric neutrino is $10-30 \%$ higher than that of anti-neutrinos; consequently, a net effect is detectable.

In practice, one needs to take into account the telescope's ability to measure the neutrino energy, the neutrino direction and to identify the neutrino flavour. Depending on the neutrino flavour, two main types of event topology are observed by the detector; Charged-Current (CC) muon neutrino interactions lead to a track-like topology, while CC electron neutrinos (and NeutralCurrent (NC) interactions of all flavours) lead to a cascade-like topology.

A first estimation of the sensitivity of ORCA to the NMO, as well as other oscillation parameters have been recently published in the Letter of Intent (LoI) of KM3NeT [3]. The main results, based on an intermediate design and first available algorithms are summarized in section 5, while sections 2 and 3 introduce the updated detector geometry and new performances in terms of triggering and reconstruction, respectively, and section 4 summarizes the classification algorithm used in the LoI.

\section{The KM3NeT detector}

The ORCA detector will be realised at the French KM3NeT site, off Toulon, at a depth of $2400 \mathrm{~m}$. The design consists of 115 flexible strings anchored on the seabed, and equipped with photo-sensors detecting the Cherenkov light resulting from neutrino interactions in the vicinity of the detector. The photo-sensors are housed within glass spheres (digital optical modules - DOMs) that host 31 three-inch photomultiplier tubes (PMTs). The strings, comprising 18 DOMs each, are connected to junction boxes that provide connections for power and data communication. 
The simulations performed for the LoI were based on a dense geometry with $20 \mathrm{~m}$ spacing between strings and $6 \mathrm{~m}$ between DOMs. This geometry served as a basis for optimising the vertical spacing by masking some of the DOMs. This procedure yielded an optimal spacing of $\simeq 9 \mathrm{~m}$ with the masking of every third DOM on each DU alternating vertical spacings of $6 \mathrm{~m}$ and $12 \mathrm{~m}$. Additional constraints from the deployment procedure resulted in a horizontal spacing increase to about $23 \mathrm{~m}$, easier to implement. The differences between the LoI based geometry and the new geometry are summarized in Table 1. The new detector configuration is about 1.4 times larger in volume than the LoI ( $9 \mathrm{~m}$ vertical spacing) one, with the same number of DOMs.

New simulations have been launched with this new geometry that incorporates a realistic arrangement of the future strings on the seabed, including the uncertainty on the positioning accuracy.

\begin{tabular}{|c|c|c|}
\hline Geometry & Vertical spacing (between DOM) & Horizontal spacing (between strings) \\
\hline \hline LoI-based [3] & $9 \mathrm{~m}$ on average with alternate $6 \mathrm{~m}$ and $12 \mathrm{~m}$ & $20 \mathrm{~m}$ \\
\hline New & $9 \mathrm{~m}$ & $23 \mathrm{~m}$ \\
\hline
\end{tabular}

Table 1: Typical distances between the detector elements for the geometry used for the LoI [3] and the updated detector configuration. Both detector geometries contain the same total number of DOMs.

Neutrino induced interactions have been generated with a software package based on the widely used GENIE [4] neutrino event generator. Electron and muon neutrino events are weighted to reproduce the conventional atmospheric neutrino flux following the Bartol model [5]. All particles emerging from a neutrino interaction vertex are propagated with a GEANT4 based software generating Cherenkov light from primary and secondary particles in cascades and simulating detected photons (hits) while taking into account the light absorption and scattering in water as well as the DOM and PMT characteristics. The background due to down-going atmospheric muons is generated with the MUPAGE program [8]. In order to reproduce the randomly distributed background PMT hits due to the Cherenkov light from electrons induced by decays of ${ }^{40} K$, single photoelectron hits can be added to the hits induced by charged particles inside a chosen time window. Also the hits in coincidence due to ${ }^{40} K$ between two PMTs inside the same DOM are taken into account. An uncorrelated hit rate of $10 \mathrm{kHz}$ per PMT and time-correlated noise on each DOM $\left(500 \times 10^{2-n}\right.$ Hz for $n$-fold coincidences) was added. These are rather conservative values, compared to the first in-situ measurements [9].

\section{Trigger and reconstruction improved performances}

\subsection{Triggering strategies}

Previous algorithms (used to produce the LoI results) used clusters of time coincident recorded photons on two PMTs of the same DOM (such coincidences are referred to as L1 hits) to match trigger conditions. For the cascade trigger algorithm, 3 time-correlated L1 hits within a sphere of $43 \mathrm{~m}\left(D_{\max }\right)$, and for the track trigger 4 time-correlated L1 hits within a tube of $39 \mathrm{~m}$ radius $(R)$ around the track were required. The distance parameters have been retuned (now: $D_{\max }=52 \mathrm{~m}$, $R=43 \mathrm{~m})$ to the new detector geometry such that a $20 \mathrm{~Hz}(10 \mathrm{~Hz})$ random noise trigger rate are allowed for the cascade (track) trigger to account for the larger horizontal separation of detection strings. 

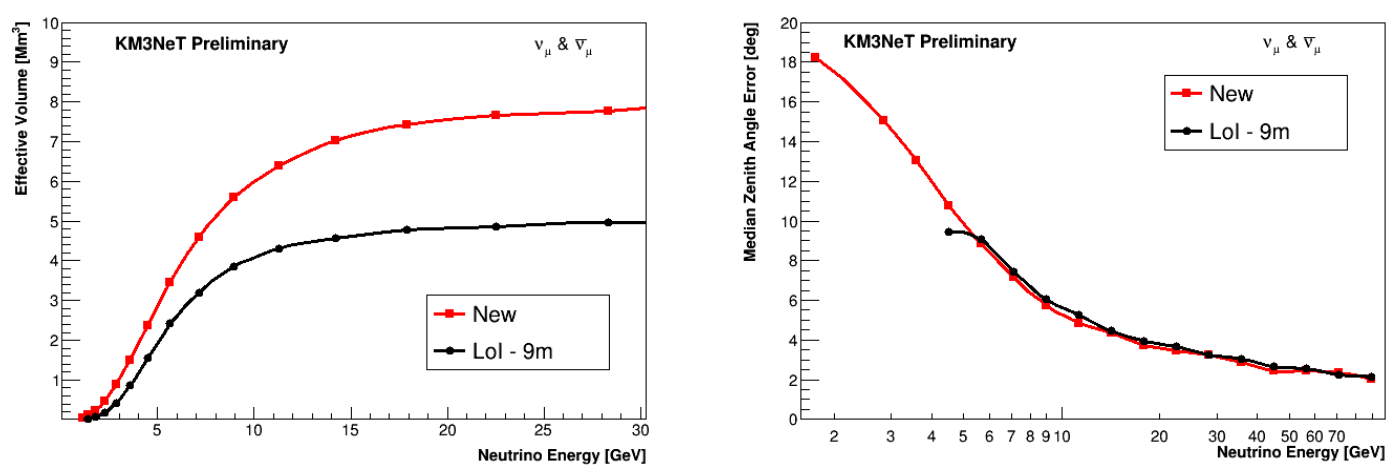

Figure 1: The effective volume (left) and median zenith angle resolution (right) in the track channel shown for the new and LoI detector layouts, with and without the latest update to the track reconstruction algorithm and trigger.

More importantly, a new trigger has been developed for ORCA which not only includes causally-connected L1 hits but is based on single hits (L0 hits) in the vicinity of an L1 hit that are causally connected among each other. Allowing also for $20 \mathrm{~Hz}$ random noise trigger rate, 7 additional L0 hits within a $D_{\max }$ of $47 \mathrm{~m}$ are required to fire the trigger. This new trigger significantly increases the effective volume near the low-energy threshold of ORCA and in the region sensitive to the mass ordering, that is in the few-GeV energy range. These additional triggered events required an update of the subsequent reconstruction algorithms.

\subsection{Track channel}

The track reconstruction proceeds through consecutive fitting iterations, each using the result of the previous one as starting point. Each fitting stage improves the result, but the last fit produced, that provides the most accurate result, works well only if the input parameters of the muon track are not too far from the true track parameters. Improvements have been achieved modifying the pre-fit stage where the scanning angle has been increased yielding a larger number track hypotheses to be reconstructed. The net improvements, in terms of effective volume can be seen in figure 1 (left). The increase in effective volume at $E>7 \mathrm{GeV}$ is primarily caused by the new, sparser detector geometry. Below $7 \mathrm{GeV}$, the change is dominated by the improved trigger efficiency and changes to the reconstruction algorithm.

The new geometry and trigger both caused the resolution to deteriorate slightly, but this was counterbalanced by improvements to the algorithm, as can be seen in figure 1 (right).

\subsection{Cascade channel}

In the cascade reconstruction, the main changes imposed by the new trigger strategy were applied in the initial hit selection stage and at the level of pre-fits aiming at localising the interaction vertex. The reconstruction now accommodates the newly gained low-energy events with a good efficiency and accuracy.

The overall performance improvements in the cascade channel can be appreciated from figure 2. For what concerns the effective volume, the increase at high energy, in the plateau regime, 

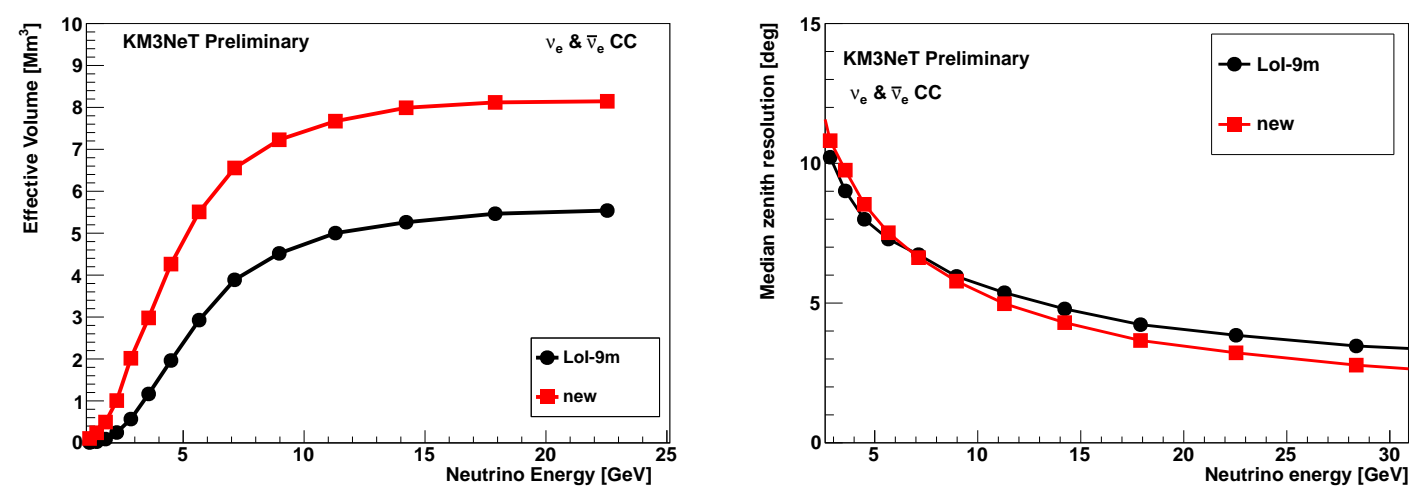

Figure 2: The zenith resolution (right) and effective volume (left) in the cascade channel shown for the current and LoI detector layouts, with and without the latest update to the cascade reconstruction algorithm and trigger.

arises from the larger and sparser detector geometry. A consequence of the improved reconstruction efficiency is a shift of the detection threshold to smaller neutrino energies, while still satisfying the bandwidth requirements of the data acquisition system (i.e. the trigger rate due to pure noise is smaller than the irreducible trigger rate due to atmospheric muons). The presence of extra low energy events does not significantly deteriorate the direction resolution, implying that the additional events can provide useful information for the physics studies. A worsening in the energy resolution is nevertheless observed as expected from lower energy (fainter) events. Despite a slightly worse energy resolution, a simplified analysis estimates a $3 \sigma$ significance on the NMO can be obtained faster than what is stated in the LoI. However, a robust estimation of the overall gain can only be achieved after the full implementation of the new analysis chain, including the classification of track-like and cascade-like events and the background rejection. This is still ongoing work. Hereafter, we describe the results achieved for the LoI, which do not include the improvements mentioned above.

\section{Classification of tracks and cascades and background rejections}

The determination of the NMO requires a precise estimate of the neutrino energy and zenith angle and a high-purity event sample with discrimination between neutrino flavours. In order to optimally exploit the information imprinted in the light emission of the events, several machine learning algorithms, so called classifiers, have been evaluated. Finally, a classification algorithm known as random decision forest (RDF) has been used in the LoI [3].

The algorithm is conceived with the distinction between three classes of events in mind. These classes are "atmospheric muons", "cascade-like" and "track-like" neutrino events. The atmospheric muon event class is induced by the passage of downward-going muons from cosmic-ray air showers which are misreconstructed as upward-going. Track-like events are those that are induced by CC muon neutrino interactions, having the signature of a straight track passing through or nearby the instrumented volume. Finally, cascade-like events are those coming from all other neutrino 

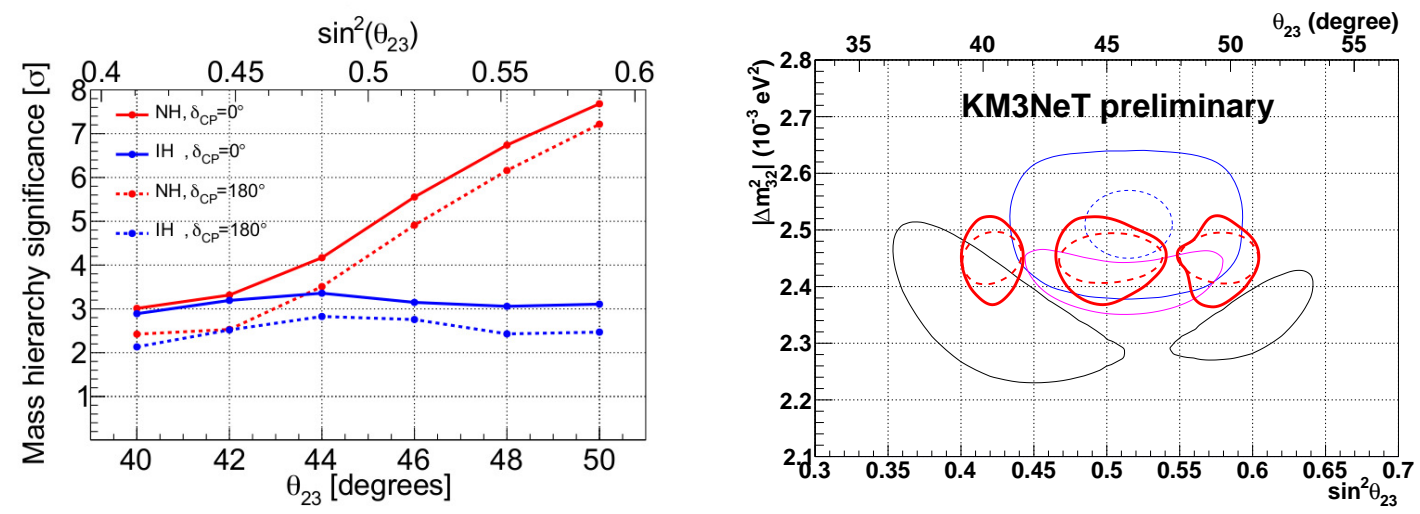

Figure 3: Left: sensitivity to the neutrino mass ordering for the LoI $9 \mathrm{~m}$ spacing configuration [3], including a fit of $\theta_{23}, \Delta M^{2}, \delta_{C P}$ and five other systematics (see text). The plot shows its dependency on $\theta_{23}$ for two values of $\delta_{C P}$ after three years of operation. Right: measurement precision in $\Delta M^{2}$ and $\sin ^{2} \theta_{23}$ after three years of data taking with ORCA with (solid red) and without (dashed red) energy scale uncertainty for three test points compared to present results from MINOS (black) and T2K (blue) and predicted performance of NOvA (magenta) and T2K (blue, dashed) in 2020. All contours are at $1 \sigma$. The plot assumed a NO scenario. Those results are conservative as improvements are expected with the implementation of new algorithms and a more realistic design of the detector.

interaction channels and flavours: all NC interactions and the CC interactions of electron and tau neutrinos.

The fraction of correctly classified events reaches $\sim 60 \%$ for $v_{\mu}, \sim 80 \%$ for $\bar{v}_{\mu}$, and $\sim 90 \%$ for $v_{e}$ and $\bar{v}_{e}$ events in the energy range relevant for the NMO measurement.

\section{Sensitivity to the neutrino mass ordering and oscillation parameters}

KM3NeT-ORCA's data will consist of observed event rates as a function of the reconstructed neutrino energy and zenith angle. By comparing these to the expected rates it will be possible to distinguish between the two mass ordering cases. The sensitivity to the mass ordering is calculated using Log-Likelihood Ratio (LLR) distributions from pseudo-experiments, while profiling nuisance parameters from interaction rate uncertainties (normalization, $\mathrm{NC}$ scale, energy slope, $v / \bar{v}$ ratio and $v_{\mu} / v_{e}$ ratio) and oscillation parameter uncertainties $\left(\left|\Delta m_{31}^{2}\right|, \theta_{23}, \theta_{13}\right.$, and $\left.\delta_{C P}\right)$.

The final figure of merit is the median significance, computed by comparing LLR distributions for true NO and true IO pseudo-experiments. Figure 3 (left) shows the mass ordering significance plot as published in the LoI [3] (they do not yet account for the improvements presented in section 3). The expected significance depends strongly on the true value of $\theta_{23}$ and to a lesser extent on $\delta_{C P}$. Without CP-violation, the NMO can be measured with more than $3 \sigma$ in three years at the current world best fit values of $\theta_{23}$.

Beyond the measurement of the NMO, KM3NeT-ORCA will also be able to measure precisely the dominant parameters responsible for oscillations at the atmospheric scale, $\Delta m_{31}^{2}$ and $\theta_{23}$. A $\chi^{2}$ minimization is performed while profiling over the same parameters in the NMO analysis, with the exception of $\theta_{13}$ which is fixed at its true value in simulations along with $\theta_{12}$, and $\Delta m_{21}^{2}$. All other eight parameters are fitted without any priors. Optionally an energy scale shift is implemented as 
an additional systematic uncertainty. Both $\Delta m_{31}^{2}$ and $\theta_{23}$ are determined under the assumption that the correct NMO has been already identified. The $1 \sigma$ contours obtained after three years of data taking for three test points are shown in figure 3 (right).

Additionally, KM3NeT-ORCA will be able to probe the appearance of tau neutrinos and make use of the MSW effect to perform Earth tomography studies, as discussed in [6] and [7].

\section{Sterile neutrinos and non-standard interactions}

Atmospheric neutrinos provide a wide range of energies and baselines (equivalent to direction), which can be used to constrain systematic effects in the regions where matter effects are not expected to occur. This same methodology can be employed to also search for physics beyond the Standard Model, by looking for distortions characteristic of resonant effects in particular models.

The new physics models studied here are phenomenological extensions of the standard neutrino oscillation picture. In the case of Non-Standard Interactions (NSI), the interaction Hamiltonian of the neutrino system is modified into an arbitrary unitary form motivated by the potential existence of new neutrino interactions that behave as four-fermion point interactions at low energies. The effect may be modelled with a set of 3 real parameters $\left(\varepsilon_{\alpha \alpha}\right)$ and 3 complex parameters $\left(\varepsilon_{\alpha \beta}\right)$ representing different neutrino flavour couplings. In the sterile neutrino case, the Hamiltonian is extended to four neutrinos. The 4th neutrino flavour is assumed not to interact in any way, yielding an effective potential difference between the sterile and active flavours due to NC interactions. Detailed reviews of these models can be found in references [10] and [11].

Preliminary sensitivities to some NSI and sterile neutrino parameters are presented. Figure 4 shows the expected sensitivities of the ORCA detector to sterile neutrinos in the $\left|U_{\mu 4}\right|^{2}-\left|U_{\tau 4}\right|^{2}$ parameter space and to NSI in the $\varepsilon_{\tau \tau}-\left|\varepsilon_{e \tau}\right|$ parameter space. For the results including systematics, the same set of systematic parameters of the mass ordering analysis are profiled over, with the exception of the energy slope uncertainty, and the $\theta_{13}$ and $\delta_{C P}$ oscillation parameters which are kept fixed at their true values. The impact of these parameters are under study. The sensitivities obtained improve on current limits by about an order of magnitude in the NSI parameters and about a factor of 2 in the $\left|U_{\tau 4}\right|$ mixing. In the case of sterile neutrinos, sensitivities were computed for $\Delta m_{41}^{2}=0.3 \mathrm{eV}^{2}$, but the sensitivity is expected to be valid for any $\Delta m_{41}^{2} \gtrsim 0.1 \mathrm{eV}^{2}$.

\section{Outlook}

The KM3NeT project is on the French roadmap of Research Infrastructures and was recently selected for the 2016 roadmap of the European Strategy Forum on Research Infrastructures (ESFRI). It is also strongly supported by the Astroparticle Physics European Consortium (APPEC) roadmap. The full detector could be completed by mid-2020. The projected sensitivities guarantee at least a $3 \sigma$ median significance determination of the NMO after 3 years of operation, in addition to competitive measurements of other oscillation parameters.

\section{References}

[1] A. Heijboer for the ANTARES and KM3NeT Collaborations, KM3NeT Physics and Status, this issue. 

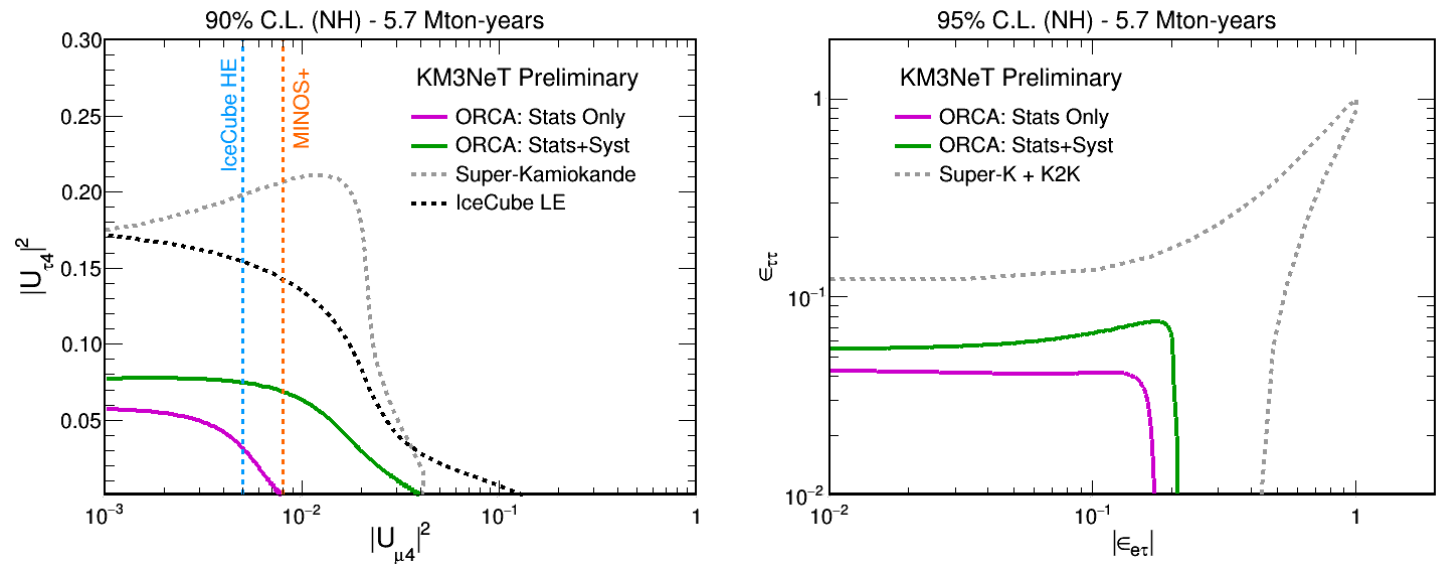

Figure 4: Sensitivity to sterile neutrinos (left) and NSI (right) for the LoI $9 \mathrm{~m}$ spacing configuration, including a fit of $\theta_{23}, \Delta M^{2}$, and four other systematics (see text). Sensitivities are for one year of operation time. For comparison, current limits on sterile neutrino mixing from Super-Kamiokande [12], IceCube [13], and MINOS+ [14] are shown as well as limits on NSI from a Super-Kamiokande and K2K analysis [15].

[2] E. K. Akhmedov, S. Razzaque and A. Y. Smirnov, Mass hierarchy, 2-3 mixing and CP-phase with huge atmospheric neutrino detectors, JHEP 02, 082 (2013). Erratum: JHEP 07, 026 (2013).

[3] S. Adrián-Martínez et al., Letter of Intent for KM3NeT 2.0, J. Phys. G, 43 (8), 084001, (2016).

[4] C. Distefano, gSeaGen: a GENIE-based code for neutrino telescopes, EPJ Web Conf. 116, 08001 (2016)

[5] G. D. Barr et al., A three-dimensional calculation of atmospheric neutrinos, Phys. Rev. D70, 023006 (2004).

[6] S. Hallman for the KM3NeT Collaboration, Tau-neutrino appearance with KM3NeT/ORCA, this issue.

[7] S. Bourret for the KM3NeT Collaboration, Neutrino Oscillation Tomography of the Earth with KMBNeT-ORCA, this issue.

[8] Y. Becherini et al., A parameterisation of single and multiple muons in the deep water or ice, Astropart. Phys. 25, 1 (2006).

[9] S. Adrián-Martínez et al., Deep sea tests of a prototype of the KM3NeT digital optical module, Eur. Phys. J. C 74:3056 (2014).

[10] T. Ohlsson, Status of non-standard neutrino interactions, Rep. Prog. Phys. 76, 044201 (2013).

[11] K. N. Abazajian et al., Light Sterile Neutrinos: A White Paper, arXiv:1204.5379 [hep-ph] (2012).

[12] Super-Kamiokande Collaboration, Limits on sterile neutrino mixing using atmospheric neutrinos in Super-Kamiokande, Phys. Rev. D91, 052019 (2015).

[13] IceCube Collaboration, Searches for Sterile Neutrinos with the IceCube Detector, Phys. Rev. Lett. 117, 071801 (2016); Search for sterile neutrino mixing using three years of IceCube DeepCore data, Phys. Rev. D95, 112002 (2017).

[14] W. Flanagan, MINOS and MINOS+ results, Tamura Symposium 2017.

[15] A. Friedland and C. Lunardini, Test of tau neutrino interactions with atmospheric neutrinos and K2K data, Phys.Rev. D72, 053009 (2005). 